\title{
Towards radiation transport modelling in divertors with the EIRENE code
}

\author{
D Reiter ${ }^{1}, \mathbf{S}$ Wiesen ${ }^{1}$ and M Born ${ }^{2}$ \\ ${ }^{1}$ Institut für Plasmaphysik, Forschungszentrum Jülich GmbH, EURATOM Association, \\ Trilateral Euregio Cluster, D-52425 Jülich, Germany \\ 2 Philips Research Laboratories, Weisshausstrasse 2, D-52066 Aachen, Germany
}

Received 7 February 2002

Published 6 August 2002

Online at stacks.iop.org/PPCF/44/1723

\begin{abstract}
Opacity effects, in particular of Lyman lines, in divertors are believed to be relevant for plasma spectroscopy and for the overall divertor dynamics through possible redistribution of excited hydrogen atoms and radiation losses. Quite elaborate computational radiation transport tools have been developed, specialized for numerous applications. The task in fusion research has been adaptation to fusion edge plasma conditions. In this paper, we start from an existing kinetic neutral particle code already well adapted to divertor applications, and extend this from the 'particle' simulation to an analogue 'photon gas' simulation. It is shown how this can be achieved and that a quite flexible and detailed divertor radiation transport code can conveniently be obtained. We apply this to study Lyman opacity effects on population kinetics and hydrogen divertor radiation losses.

This article was scheduled to appear in issue 7 of Plasma Phys. Control. Fusion. To access this Special issue on advanced Tokomak research in EFDAJET please follow this link: http://stacks.iop.org/0741-3335/44/7
\end{abstract}

\section{Introduction}

Opacity effects, in particular on the resonance Lyman lines, have already been observed experimentally and computationally for dense divertor plasma conditions in various Tokamaks, e.g. Alcator C-Mod [1], in MARFEs [2], and they may be quite relevant for the operational window for ITER-FEAT [3] under the anticipated normal operation under 'partially detached' conditions. For example, volume recombination, a crucial effect in detached divertor states, is expected to be reduced significantly by resonant photon re-absorption, while ionization will be enhanced. Previous computational assessments have usually been based upon highly idealized, often zero-dimensional approximations (so called optical escape factors in collisional-radiative models) [4] or, at best, one-dimensional approximations [5,6]. Due to the spatial gradients of neutral gas profiles in divertors, the various line-broadening mechanisms and the often kinetic (non-fluid) properties of the neutral gas components in divertors a quantitative bookkeeping 
of radiation processes seems to require Monte Carlo photon gas simulations also for dense divertors and in MARFEs. Such procedures are already well established in many fields, for example, in astrophysics, for low- and high-pressure discharges used for lighting purposes, laser-produced plasmas, plasma vapour shielding and many others. The effort to adapt these specialized codes to typical fusion edge plasma conditions, especially configurational details, is quite considerable, but some steps have already been undertaken, for example, with the CRETIN code [7].

In this paper, we take the opposite approach: in a current joint effort with lighting industry, the EIRENE neutral particle transport code [8], widely used in fusion edge plasma studies, is extended from it's test particle (neutrals and ions) options towards photon gas simulations. Applications are envisaged for both fusion edge plasmas and high-pressure gas discharge lamps. The radiation transfer equation is mathematically analogue to the linear Boltzmann equation for neutral particles solved by EIRENE. Because of that most of the existing coding can be used directly for photon transport problems, with only minor modifications and without large amounts of duplicating work. This is outlined in the second and third section. The relevant line emission profiles (typically: Voigt profiles) have been implemented into EIRENE, the spatial distribution of emission of radiation can be treated by the routines already in place for volume recombination processes for neutral particles. Absorption coefficients can be formulated in the same format as the present atomic and molecular databases used by EIRENE, with the energy dependency replaced by a wavelength dependency.

In sections 5 and 6 , we test the photon gas simulation by comparing our Monte Carlo solutions with semi-analytical results, such as population escape factors and in the thermodynamical limit (spectral line shapes vs Planck'ian). The 'conditional expectation estimator' technique, optional in EIRENE [8] since long, permits reduction of the Monte Carlo procedure to analytical solutions without any noise, for point sources and purely absorbing media. This allows to eliminate statistical noise even for highly opaque conditions, as long as scattering (other than stimulated emission) is neglected, which we will do in this paper. The EIRENE code has been fully parallelized for multi-processor machines, as routinely utilized in the three-dimensional stellarator applications [9]. We will, however, not use this option in this paper and all runs discussed below have been performed on an IBM RS/6000 workstation, which is more similar to the platform used in most routine B2-EIRENE applications in fusion edge plasma studies in most laboratories. In the final section, we apply the extended code to a model typical of conditions expected in the ITER-FEAT scrape off layer and divertor, from earlier B2-EIRENE simulations [10], [11].

\section{Basic linear transport equations for particles and photons}

The material in this sections can be found in many textbooks, such as [12] on the Boltzmann equation, or [13] on radiation transport in stellar atmospheres. Furthermore, in this paper we shall not deal with (numerical or statistical) approaches to the radiation transfer problem as such. For this topic see, for example, the monograph by M F Modest [14] and references therein.

Here we only give a brief 'dictionary' for translating the terminology typical of radiation transport into that of neutral particle (or also neutron) transport.

The basic quantity of interest in photon gas transport is, usually, the specific intensity (also: 'brightness') $I_{v}=I_{v}(\vec{x}, v, \vec{\Omega}, t)$. It has the dimensions: energy (time) ${ }^{-1}$ (area) $^{-1}$ (solid angle $)^{-1}$ (frequency) $)^{-1}$.

We first convert the frequency $v$ into an energy $E, E=h v$ with Planck's constant $h$. Then the velocity space coordinates of a single ray $(E, \vec{\Omega})$ can be transformed into the velocity 
vector $\vec{v}$, upon which, usually, neutral particle transport codes for fusion edge plasmas are based. In order to recover $(E, \vec{\Omega})$ from $\vec{v}$, the frequency needs to be added to the phase-space of a test particle in the case of photons, $E=h v$, whereas for neutral atoms or molecules the mass is needed: $E=m / 2\left|v^{2}\right|$. The state of a test-particle in EIRENE is given by $m, \vec{x}, \vec{v}, E$, $t$, hence, due to this 'redundancy in the dimensionality of the problem', it is suitable for both particles and photons.

Now we can identify the specific (radiation) intensity $I_{v}$ with the 'energy transport flux' $\tilde{I}=\tilde{I}(m, \vec{x}, \vec{v}, E, t)$,

$$
\tilde{I}=E v f(m, \vec{x}, \vec{v}, E, t)
$$

and $f$ is the usual particle distribution function used in neutral particle transport, and $v=c$, the vacuum speed of light. For technical reasons, the Monte Carlo code EIRENE (loc.cit.) solves the linear Boltzmann equation for the transport flux $\Phi(=v f)$ as dependent variable, rather than for the distribution function $f$ itself. It provides responses $\langle g, \Phi\rangle$ for arbitrary (problem specific) 'detector functions' $g(m, \vec{x}, \vec{v}, E, t)$. The brackets are to be understood as phase-space integrals of $g \Phi$ and hence are cell averages with the choice of 'cells' (small sub-domains of the phase-space) determined from the resolution needed for any particular transport problem.

Choosing $g=E$, we see that kinetic neutral particle transport codes for $\Phi$ also solve the radiation transfer equation for $\tilde{I}$ as a special case.

For example the photon flux $F$ across a surface normal to the ray directly corresponds to the (scalar) transport flux $\Phi$ and is the first angular moment of the specific intensity $I$ :

$$
F=\int I \cos (\theta) \mathrm{d} \Omega=\int I \cos (\theta) \sin (\theta) \mathrm{d} \theta \mathrm{d} \varphi
$$

All responses computed from the neutral particle simulation codes for current plasma edge simulations tools as, for example, B2-EIRENE [15], such as ionization rates, surface fluxes, plasma cooling rates or momentum exchange rates (neutral-plasma friction), can, without any modification of the coding, now also be obtained for the photon gas. The complication of nonlinearity in some collision integrals, resulting from the mutual influence of the radiation field and the exited state population of neutral particles can be dealt with by the same iteration procedure as it is already available for neutral-neutral particle collisions [16].

The volumetric source function for neutral particles, e.g. due to plasma recombination, can be re-interpreted as the spontaneous photon emissivity for the case of radiation transfer. The external volumetric source for neutral atoms, for example, due to recombination of plasma electrons and ions, reads

$$
S_{\mathrm{rec}}(\vec{r}, \vec{v})=n_{\mathrm{e}}(\vec{r}) n_{\mathrm{i}}(\vec{r})\left\langle\sigma v_{\mathrm{e}}\right\rangle_{\mathrm{rec}}\left(T_{\mathrm{e}}, n_{\mathrm{e}}\right) \tilde{f}_{\mathrm{i}}(\vec{v})
$$

The product of the first 3 factors is the recombination rate, with $\langle\cdots\rangle_{\text {rec }}$ the multi-step recombination rate coefficient [17]. $\tilde{f}_{\mathrm{i}}$ is the normalized ion distribution function, which is also used as initial distribution in velocity-space of atoms after recombination. It is, usually, a drifting Maxwellian. The corresponding photon emission term $j_{v}$ is given below, but it is obvious that only the recombination rate has to be replaced by the (spontaneous) emission rate, and that the normalised velocity distribution $\tilde{f}_{\mathrm{i}}(\vec{v})$ is replaced by the normalized emission line shape function $\phi(v)$, determined by the line-broadening mechanisms taken into account.

Absorption and scattering (excluding stimulated scattering) are entirely analogous in the two cases of photon gas and neutral gas simulation. Redistribution due to scattering of photons in the volume is described by the so called 'phase function'; in case of neutral particles, this same quantity is usually referred to as 'scattering kernel'. The extinction coefficient $\chi(\vec{x}, v, t)$ (also: 'opacity', or 'total absorption coefficient') in radiation transport is 
the inverse of the photon mean free path, and, hence, the same quantity as the 'total macroscopic cross section' $\Sigma_{t}(\vec{x}, E, t)$ (Dimension: (length) $)^{-1}$ ) in neutron and neutral gas transport terminology [8].

The full, self-consistent, Monte Carlo model for neutral and photon gas transport for divertors and MARFEs, eliminating the hitherto introduced ad hoc opacity parameters from the model, is obtained by replacing the kinetic neutral atom (ground state) transport equation (for $f_{1}$ ) now by a coupled set of three mathematically identical transport equations for groundstate atoms $f_{\tilde{1}}$, for atoms in the upper Lyman $\alpha$ state, i.e. in the $n=2$ excited state $f_{\tilde{2}}$ and for photons of the Lyman $\alpha$ line $f_{\mathrm{Ly}}$. A typical multi-species B2-EIRENE divertor plasma simulation is a kinetic multi-species problem already, with hydrogenic, helium and impurity atoms, various molecules and molecular ions, resulting in about 20 different species treated simultaneously by the Monte Carlo code. Increasing this number by 2 seems to be tolerable in terms of CPU and storage resources on current computing platforms.

\section{Reformulated ionization and recombination rates for neutral atoms}

If we include photons of a certain line (say: of the Lyman $\alpha$ line) as explicit species in the kinetic neutral gas transport model, in addition to atoms and molecules, then the effective ionizationand recombination-rate coefficients used in the particle transport model also have to be revised, because these effective (density dependent) rate coefficients already include assumptions about radiation transport. The usual assumption is either 'zero opacity', then neglecting all re-absorption processes of photons in the balance equations for the exited-state population coefficients, or perfect opacity. In this latter case, the corresponding radiative transition rates (e.g. for Lyman lines: $A_{n, 1}$ ) are set to zero. Intermediate is the concept of 'population escape factors', which are multiplicative factors to these rates $A_{\mathrm{i}, k}$ obtained from semi-analytical evaluations of photon transport in idealized geometries (cylinders, spheres, etc) and for simple one-dimensional expressions (usually: constant) of the emission and absorption profiles, see section 5 and [4].

For our combined neutral atom-photon gas simulation, we first have to eliminate the relevant transition rate (here: $A_{2,1}$ ) from the rate equations of excited-state populations, because this rate now is explicity included in the source term for the Lyman $\alpha$ photons. Doing so, the $n=2$ level of hydrogen atoms becomes 'metastable' in the collision-radiative model and has to be included as further new species in the multi-species atom-photon gas simulation. As already described in detail in [17], the usual collisional-radiative ionization-recombination balance

$$
\frac{D}{D t} n_{1}=-s n_{1}+\alpha n_{\text {ion }}+\text { o.c. }
$$

for ground-state atom density $n_{1}$ now becomes

$$
\begin{aligned}
& \frac{D}{D t} n_{\tilde{1}}=-s_{1} n_{\tilde{1}}-s_{1,2} n_{\tilde{1}}+s_{2,1} n_{\tilde{2}}+\alpha_{1} n_{\text {ion }}+\text { o.c. } \\
& \frac{D}{D t} n_{\tilde{2}}=-s_{2} n_{\tilde{2}}-s_{2,1} n_{\tilde{2}}+s_{1,2} n_{\tilde{1}}+\alpha_{2} n_{\text {ion }}+\text { o.c. }
\end{aligned}
$$

for the two effective atomic species with densities $n_{\tilde{1}}$ and $n_{\tilde{2}}$.

Here $s$ and $\alpha$ are the effective rate coefficients for ionization and recombination, respectively, (density and temperature dependent) which include the effects of the 'lumped species' (terminology from air pollution modelling), in our case of all the higher excited atoms, which travel in collisional-radiative equilibrium with the effective ground-state atoms (case 3) and effective ground-state and effective $n=2$ excited-state atoms (case 4 ), respectively. $D / D t$ 
is the total (convective) derivative, and o.c. stands for 'other collision terms', such as charge exchange, contributions from dissociating molecules, recombining molecular ions ('MAR') etc. In total, we are dealing with about 100 excited species (atomic or molecular). However, due to the very different timescales for their relaxation towards a population equilibrium consistent with the radiation field, most of the convective derivatives can be set to zero.

For example, also the MAR rate coefficient

$$
p+H_{2} \rightarrow H+H_{2}^{+}, \quad e+H_{2}^{+} \rightarrow H+H
$$

now has to be replaced by two effective MAR rate coefficients:

$$
\begin{array}{ll}
p+H_{2} \rightarrow H+H_{2}^{+}, & e+H_{2}^{+} \rightarrow H+H(n=1) \\
p+H_{2} \rightarrow H+H_{2}^{+}, & e+H_{2}^{+} \rightarrow H+H(n=2)
\end{array}
$$

The same applies for the other molecular channels leading to neutral atoms in the EIRENE model, i.e. dissociation into ground and excited states, and for the destruction of negative ions. All these effective rate coefficients have been computed on the basis of the most recent extension of the collision-radiative code from K Sawada and T Fujimoto [19,20], including 35 electronically excited singlet and 35 electronically excited triplet molecular states, the vibrationally excited molecular ground states as well as a full set of electronically excited atomic states. These multistep rate coefficients are available on the EIRENE web domain [8].

Amongst the 'other collision terms', not any longer included in the revised collisionalradiative model data for hydrogenic atoms, are now the new terms for the radiative coupling between the ground state and the $n=2$ state. These will be described in the next section. The commonly simulated 'optical thin case' (equation (3)) and the corresponding collisionalradiative effective rates are recovered from our general case (equations (4) and radiation transfer) by only retaining spontaneous emission (see (i), next section) amongst these coupling terms and by setting $(D / D t) n_{2}$ to zero. Then $n_{2}$ can be expressed as linear combination of $n_{1}$ and $n_{\mathrm{i}}$. Inserting this in the balance equation for $n_{1}$ gives equation (3), also with the same effective coefficients as in (3).

\section{Rate coefficients for the photon gas}

To describe emission and absorption processes for one spectral line microscopically, we consider two distinct energy levels in an atom with energies $E_{1}$ and $E_{2}$ (we assume $E_{1}<E_{2}$ and $\left.E_{2}-E_{1}=h v_{0}\right)$ and statistical weights $g_{1}$ and $g_{2}$. Then three processes can be identified:

(a) Spontaneous emission: this is determined by the Einstein A-coefficients, i.e. by $A_{21}=$ transition probability per unit time for spontaneous emission (units $\mathrm{s}^{-1}$ ). These coefficients are 'rates' in the transfer equation, or, if divided by the upper level density: 'rate-coefficients' For the Lyman $\alpha$ line considered below we have $g_{1}=2, g_{2}=8, A_{2,1}=4.699 \times 10^{8} \mathrm{~s}^{-1}$.

The line profile $\phi(v)$ needed to determine the frequency of test-photons in the simulation is given by convoluting the line profiles for all the individual broadening mechanisms taken into account (see also next item: absorption). Random sampling of the frequency from the convolution integral, if more than one broadening effect is included, can be done simply by sampling, independently, from the individual line profiles and then adding the random frequencies. This is due to a corresponding general law for sampling from convoluted distributions. Currently we have implemented Lorentz and Doppler profiles, hence also Voigt profiles.

(b) Absorption: due to the uncertainty principle for the energy levels (natural broadening) and other broadening processes like Doppler shift, the differences between the energy levels 
are not sharp. Photons with frequencies in the vicinity of the line centre at $v_{0}$ can be absorbed by ground-state atoms. This process leads to a loss of ground-state atoms travelling in a background of photons, and hence appears as an additional sink (volumetric absorption process) in the equation for $f_{\tilde{1}}$, and as additional source in the equation for $f_{\tilde{2}}$. The corresponding rate involves the line profile function $\phi(v)$. This function $\phi(v)$ is peaked at the line centre and is normalized by

$$
\int_{0}^{\infty} \phi(v) \mathrm{d} v=1
$$

The probability for absorption per unit time for ground-state atoms is then $r_{\tilde{1}}=B_{1,2} \bar{J}$, dimension: time $^{-1}$, where

$$
\bar{J}=\int_{0}^{\infty} J_{v^{\prime}} \tilde{\phi}\left(v^{\prime}\right) \mathrm{d} v^{\prime}
$$

is the frequency integrated mean intensity, $v^{\prime}$ is the frequency Doppler-shifted due to the velocity of the impacting atom, $\tilde{\phi}$ is the line profile function with the Doppler broadening contribution removed. Finally

$$
J_{v}=\frac{1}{4 \pi} \int I_{v} \mathrm{~d} \Omega
$$

is the zeroth angular moment of $I_{v} . B_{1,2}$ is the so called Einstein B-coefficient for absorption. If, instead, a Monte Carlo test-photon is travelling in a bath of (background) ground-state atoms, the same process leads to a loss of the photon (and again: a new $n=2$ atom), with a rate

$$
r_{\mathrm{Ly}, \mathrm{abs}}=c \frac{h v}{4 \pi} \phi(v) n_{\tilde{1}} B_{1,2}
$$

Here, $\phi(v)$ includes the Doppler contribution from the ground-state atoms with temperature $T_{\tilde{1}}$.

(c) Stimulated emission: distinct from neutral atoms, the photons are Bosons. Hence, both the processes of emission and scattering are enhanced by the number of photons already in the final state ('stimulated' or 'induced' emission and scattering). We do not treat scattering processes (i.e. processes with finite momentum transfer) of photons with the atomic gas or the plasma at all here, because these are irrelevant for the typical energies in fusion edge plasmas. Therefore, the complication resulting from 'stimulated scattering', which would render the radiation transfer equation itself nonlinear, does not arise here.

'Stimulated emission' is treated as if it were a scattering event in the Monte Carlo framework, in the sense described next. It is the only 'true' scattering process included in the photon simulations. It's rate-coefficient is related to the $B_{2,1}$ Einstein coefficient. A photon incident on a background $n=2$ atom produces a second photon, with a rate (dimension: time $^{-1}$ )

$$
r_{\text {Ly,stim }}=c \frac{h v}{4 \pi} \phi(v) n_{\tilde{2}} B_{2,1}
$$

This scattering is 'coherent': it does not alter the frequency, nor the direction of a test photon. In neutron transport, such collision terms are referred to as delta-scattering or pseudo-collisions. Since the number of test-particles is altered, this corresponds, in this regard, to fission (neutrons) or, for example, dissociation (neutral particles). Such processes are particularly trivial to include in Monte Carlo simulations as they only alter the weight of a test-flight along the trajectory. In radiation transport simulations, the corresponding scattering rates are usually condensed with the absorption-rate into one effective absorption rate, i.e.

$$
r_{\mathrm{Ly}, \mathrm{eff}}=r_{\mathrm{Ly}, \mathrm{abs}}-r_{\mathrm{Ly}, \mathrm{stim}}
$$


can be used as absorption rate for test photons in Monte Carlo solutions of the radiation transfer equation.

Details about these three processes, in particular the Einstein relations between them, can be found in many textbooks. For the particular format needed to implement these as special case into existing neutral particle transport models, we refer to the EIRENE web-domain [8].

\section{Population escape factors}

As a first check for the validity of the photon-transport simulations with EIRENE, we compare our Monte Carlo results with semi-analytical so-called 'population escape factors'. There are several different definitions of these factors in the literature. The one used here (and in the semi-analytical evaluation of Behringer, [4]) is given in [18]. We consider the population escape factor $\Theta_{p}$ from [4], defined as

$$
\Theta_{\mathrm{p}}=1-\frac{\int_{\Omega} \int_{\text {line }} \chi_{\nu}(x) I_{v}(x, \Omega) \mathrm{d} \nu \mathrm{d} \Omega}{4 \pi \int_{\text {line }} j_{v}(x) \mathrm{d} \nu}=1-\frac{G}{E}
$$

where $\chi_{v}(x)$ is a spatially varying absorption coefficient for photons, $j_{v}(x)$ the emission coefficient, where e.g. $j_{v}=n_{2} A_{2,1} \phi_{v}$ for the Lyman- $\alpha$ line, $I_{v}$ the spectral intensity and $\mathrm{d} \Omega$ the solid angle element. Note that the emission and absorption profiles are included in $j_{v}$ and $\chi_{\nu}$, respectively. The numerator $G$ is the number of absorption processes in the plasma at position $x$ and the denominator $E$ is the number of emission processes (both per unit volume and per time).

This correction factor for Einstein coefficients in collisional-radiative models is a function of the 'optical thickness' $\tau$, or, if the profile function $\phi(v)$ is given, of $\tau_{0}=\chi\left(v_{0}\right) b$. $b$ is the characteristic length in the plasma and $\chi\left(v_{0}\right)$ is the absorption coefficient in the line centre. $\Theta_{p}(x)$ furthermore depends upon the geometry. It is not a local quantity, since the source of photons is distributed in the entire volume. This definition has to be carefully distinguished from the 'line escape factors', which are more relevant for spectroscopic applications, but, due to a spatial delta distribution in the source, provides only a trivial test for a Monte-Carlo evaluation, if the conditional expectation estimators are used ( [8]).

In order to reproduce the semi-analytical population escape factors for Lyman $\alpha$, Lyman $\beta$, Lyman $\gamma$ and Lyman $\delta$ lines of hydrogen given in [4] we simulate a homogeneous hydrogen plasma in a cylinder of radius $b=5 \mathrm{~cm}$. The 'plasma' consists of hydrogen in the ground-state $n=1$ and upper excited states $n=2,3, \ldots$ for the Lyman lines. The parameters chosen here are $n_{\mathrm{H}}=10^{14} \mathrm{~cm}^{-3}$ and $T_{\mathrm{H}}=1 \mathrm{eV}$. The density of the excited-state atoms was set according to the LTE value. However, this absolute emitter density is irrelevant for these factors under the conditions investigated here, because the contribution of stimulated emission to Lyman lines turns out to be negligible at such low temperatures.

Emission and absorption was modelled as described above, with Doppler broadening (again $1 \mathrm{eV}$ ) as the only line-broadening mechanism. Figure 1 compares the numerical results from [4] with our Monte Carlo calculations for the Lyman lines, both taken on the axis of the cylinder. The population escape factors $\Theta_{\mathrm{p}}$ for Lyman $\beta, \gamma, \delta$ can perfectly be matched . The result for Lyman $\alpha$ has the largest statistical noise $(0.2 \%$ for the absorption rate on axis), due to high opacity ( $\tau_{0}$ about 27 ) for this line, but also agrees perfectly within the statistical error bars.

In order to use these factors for a divertor plasma analysis one would have to decide first which of the elementary configurations (cylinders, spheres, slab) and which of the precomputed plasma profiles (constant, parabolic etc) are closest to the real divertor conditions under 


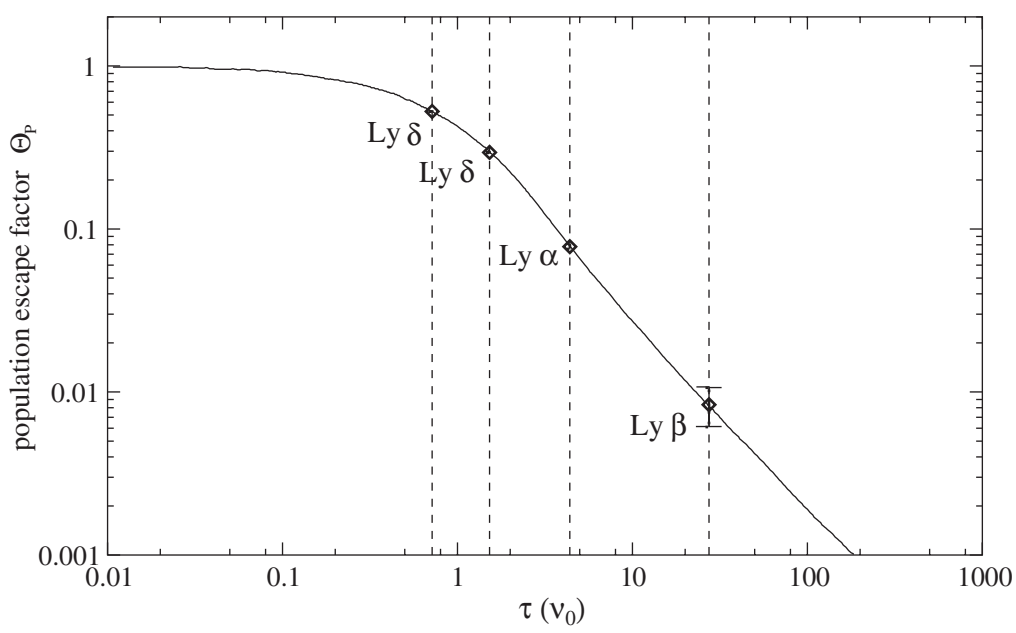

Figure 1. Population escape factors $\Theta_{p}$ for four different hydrogen Lyman lines. $\tau\left(v_{0}\right)$ is the optical depth in the line centre. Solid line: [4], symbols: EIRENE simulation. Error bars are plotted for all lines, but are visible only in the highly opaque Lyman $\alpha$ case in this scale.

investigation. Then, the collisional-radiative rates (equation (3)) have to be recomputed for each cell of the computational grid with the effective $\tilde{A}_{\mathrm{i}, k}\left(\tilde{A}_{i, k}=\Theta_{p} \cdot A_{i, k}\right)$ to derive the ionization and recombination rates. The same is achieved, however without any of these simplifications of physical or geometrical nature, by the extended Monte Carlo procedure described here, for arbitrary three-dimensional configurations, plasma and line profiles. In the last section, we will show the resulting population escape factor profile for such a 'real' ITER divertor simulation.

\section{Planck function for optically thick hydrogen Lyman $\alpha$ line}

Because only the ratio of absorbed and emitted photon numbers enter into the population escape factor, the above validation does not check absolute values of the computed specific intensity function $I_{\nu}$. This can be achieved by comparing the calculated spectral intensity with the Planck function for optically thick lines. In this optically thick limit, the correctness of a kinetic Monte Carlo scheme requires particular attention. The Planck function,

$$
B_{v}=\frac{2 h v^{3} / c^{2}}{\exp (h v / k T)-1}
$$

represents the spectral intensity $I_{\nu}$ for the special case of blackbody radiation. We use the same geometry as in the previous section, except that now we treat a finite cylinder (length: $10 \mathrm{~cm}$ ). For the hydrogen Lyman $\alpha$ line, which starts to become optically thick in our calculations at densities above $10^{14} \mathrm{~cm}^{-3}$, the spectral intensity should approach the Planck curve from below, with increasing opacity, if the upper level density (emitting species) is kept in local thermodynamic equilibrium (LTE) with the lower. Figure 2 shows Doppler broadened lines at different absorber densities in comparison to the Planck distribution. The temperature of the hydrogen gas was again set to $1 \mathrm{eV}$, the densities of the upper $n=2$ level is determined by the Saha-Boltzmann distribution with this temperature at LTE. The Planck curve in figure 2 for $T=1 \mathrm{eV}$ in the close vicinity of the Lyman $\alpha$ line, i.e. near $10 \mathrm{eV}$, is almost constant. 


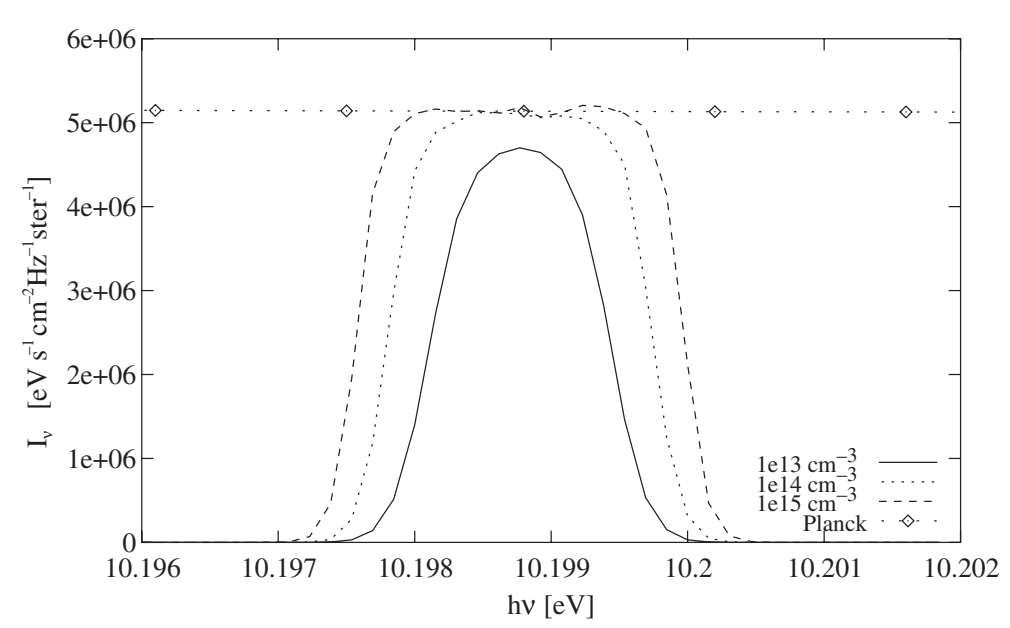

Figure 2. Intensity functions of Lyman $\alpha$ radiation escaping from a cylinder $10 \mathrm{~cm}$ in diameter, $10 \mathrm{~cm}$ in length, in comparison to Planck's distribution. The intensity is plotted for three different absorber densities $1 e 13,1 e 14$ and $1 e 15 \mathrm{~cm}^{-3}$ at $k T=1 \mathrm{eV}$. At higher densities the intensity is approximating the Planck limit and flattens in the line centre.

The expected feature of approaching the Planck curve in the limit, in which now even the photon gas in this selected line approaches the thermodynamical equilibrium, is clearly recovered. This demonstrates that even a photon gas in thermal equilibrium is exactly modelled by our kinetic Monte Carlo approach, although, of course, it is patently foolish to use a Monte Carlo code under such conditions. The future goal still must be to optimize statistical performance near this limit, if a wide range from optically thin to optically thick has to be modelled within the same case.

\section{Sample applications to ITER-FEAT conditions}

The extended EIRENE code is employed here to study the effects of Lyman opacity in divertors, with the particular conditions chosen here being taken from B2-EIRENE simulations of ITER-FEAT ( [10]). Starting point was a well-converged simulation for a V-shaped divertor configuration, reference case 463. Details about this study are given in [10], and, in a broader view, in [11]. Relevant for our particular application here is the observation that both the ion and the electron densities are still low enough to neglect Stark broadening effects.

Figures 3 and 4 display the plasma temperature and density, respectively, chosen in this sample application. A total power of $100 \mathrm{MW}$ (50\% in electrons and 50\% in ions) was assumed to flow from the core into the edge region, with a plasma density of $4 \times 10^{19}$ there. Deuteron, helium and carbon ions had been included in the B2-EIRENE plasma flow simulation, resulting in the detached divertor conditions shown here. The deuteron flow field is shown in figure 5.

Distinct from common B2-EIRENE simulations the two-dimensional grid for the plasma field (as used in figure 5) has to be supplemented by a finite element discretization of the entire 'vacuum region' between the outermost flux surface used in the plasma simulation and the vessel. This extension was already developed in [16] to allow for nonlinear neutral-neutral interactions. This option permits spatial resolution of the neutral gas (and photon gas) field also in this outer domain, and, therefore, to account for the bi-linear interaction between photons and atoms by iteration. Neutral atom- and molecule-density profiles are shown in figures 6 


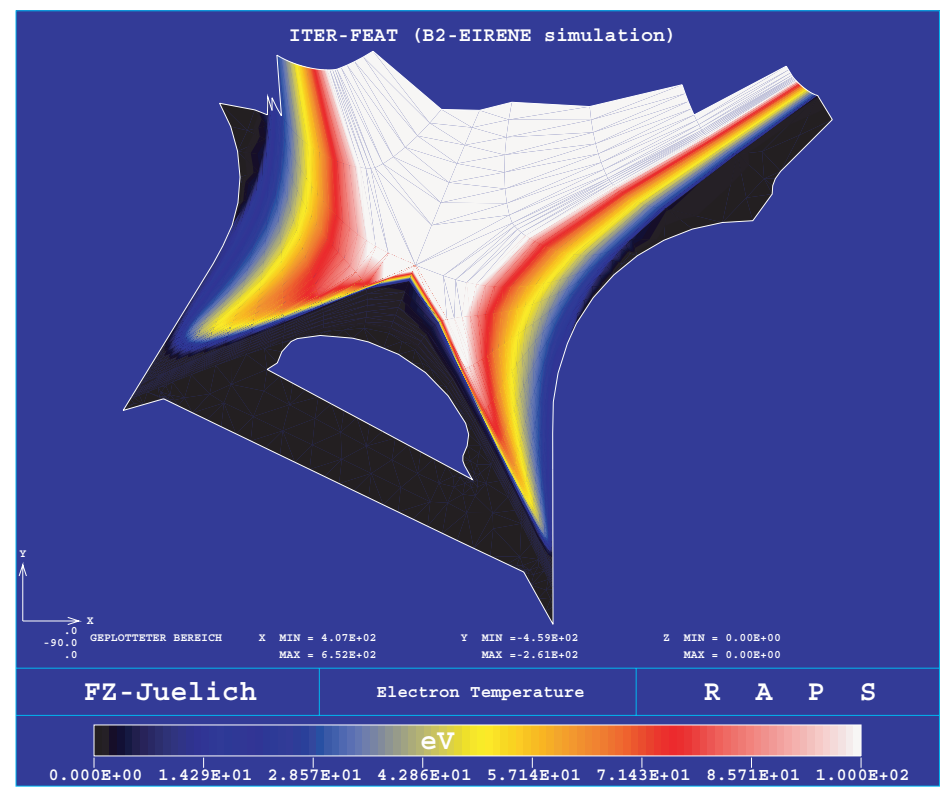

Figure 3. Electron temperature field (eV) from B2-EIRENE model. A linear scale for the colour code has been used, ranging from 0 (black) $\geqslant 100$ (white) $\mathrm{eV}$. In particular the detachment front at the inner target is clearly visible.

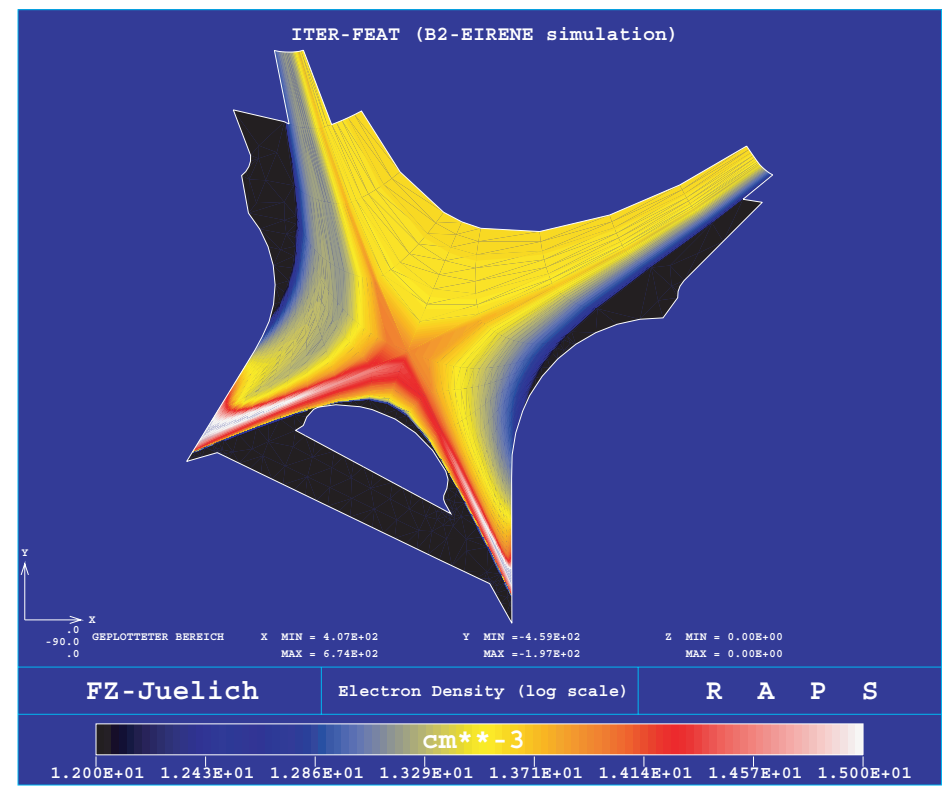

Figure 4. Electron density field $\left(\mathrm{cm}^{-3}\right)$ from B2-EIRENE model. The colour code is equidistant on a logarithmic density scale, ranging from $\leqslant 10^{12}$ (black) to $\geqslant 10^{15}$ (white).

and 7. A small pump (recycling coefficient below one) was imposed at the bottom surface between the two divertor legs, to provide sufficient helium removal.

These atom and molecule densities have been obtained with the usual optically thin approximation. To start the nonlinear iteration procedure at this point, we have derived 


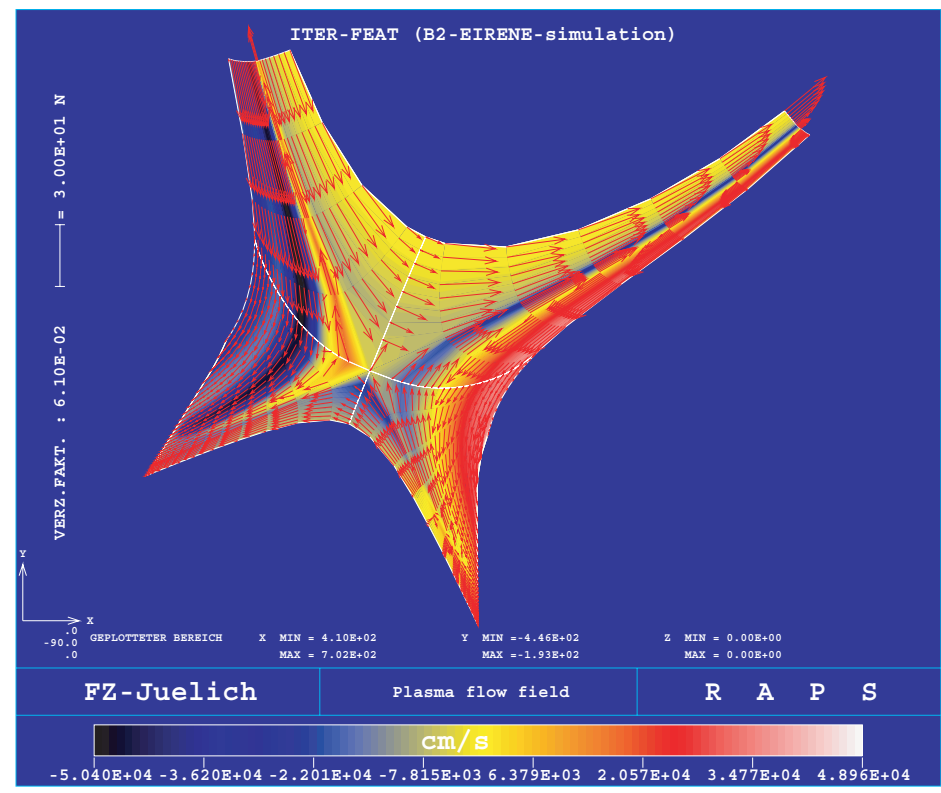

Figure 5. Deuteron ion flow field, from B2-EIRENE model. Blue background colours in outer divertor and red background colours in inner divertor indicate flow reversal.

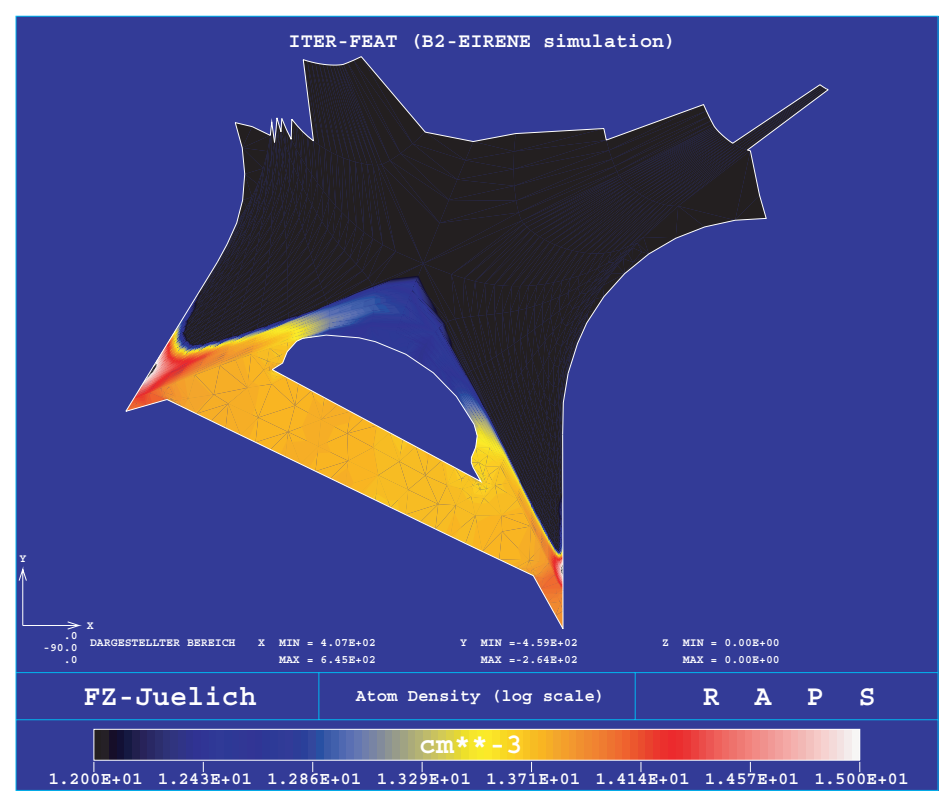

Figure 6. Neutral atom density, $\mathrm{cm}^{-3}$, log. colour scale, from original B2-EIRENE model. Finite element spatial resolution outside the plasma domain included, for nonlinear Lyman photon simulation in later iterations.

the $n=2$ excited-state density profile, from the ground-state atom density profile and the plasma density and temperature on the basis of the ordinary (optically thin) collisionradiative population coefficients taken from the EIRENE database. This provides, for a first iteration step, the volumetric source of Lyman $\alpha$ photons due to spontaneous emission. Doppler 


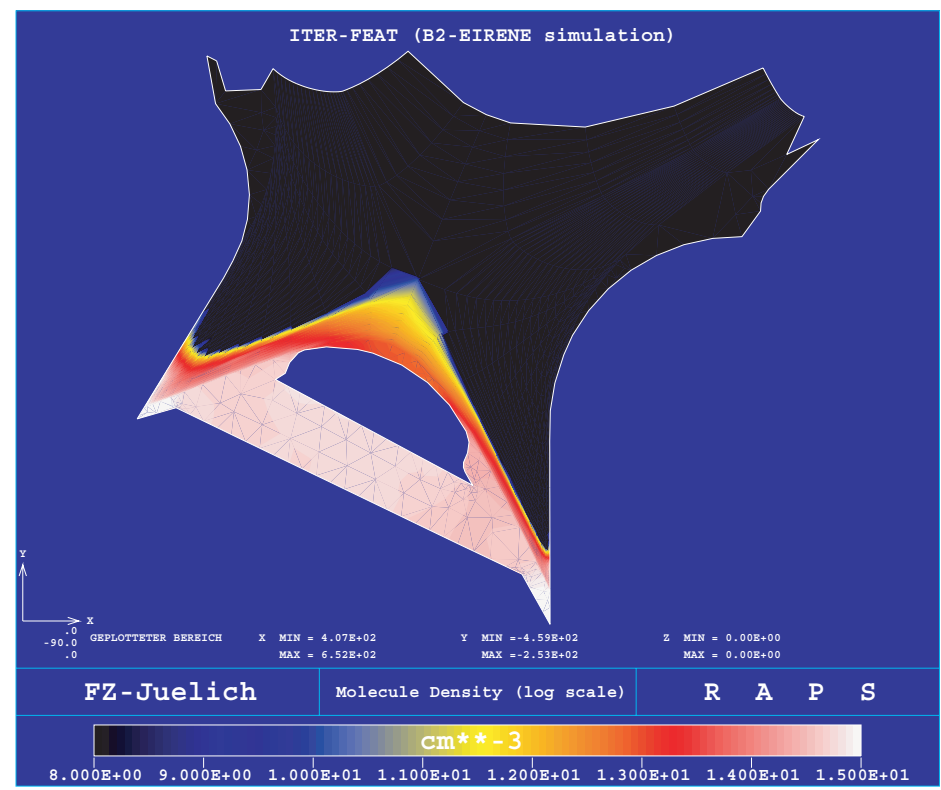

Figure 7. Same as figure 6, but $\mathrm{D}_{2}$ molecule density.

broadening is taken into account on the basis of the two-dimensional temperature field of neutral atoms (the same for ground state and excited states in this first iteration) as the only relevant line-broadening mechanism considered here. Together with this additional new species (the photons of the Lyman $\alpha$ line), the effective collisional-radiative rate coefficients for the ground state and the $n=2$ excited test particle species are modified by switching from the balance expressed in equation (3) to the balance of equation (4) in section 3.

Each individual EIRENE run then provides new solutions for the test particle fluxes $\Phi_{\tilde{1}}, \Phi_{\tilde{2}}$ and $\Phi_{\mathrm{Ly} \alpha}$, given the same quantities from the previous iteration, which define densities, temperatures and radiation field of atoms and photons, respectively, used as additional (to the plasma components) background species.

Note that this does not require any extra iterative procedure, because due to the usual (bi-linear) neutral-plasma interaction the B2-EIRENE code has to iterate anyway between neutral kinetic and plasma fluid solutions.

The results of that nonlinear neutral-photon simulation in this present paper, however, are obtained with fixed plasma profiles, i.e. without any further runs of the B2 plasma fluid code in between. Hence only the modification of the neutral gas fields due to consistent radiation transfer simulations is addressed here.

After convergence the atoms density has decreased significantly, due to re-absorption of Lyman $\alpha$ photons. This is shown in figure 8, to be compared with figure 6 . The same colour code is used in both results, and both are obtained for identical plasma and geometrical conditions. As to be expected trapping of radiation shifts the ionization-recombination balance in divertors towards a more ionizing state, by assisting excitation of atoms into the $n=2$ state, from which ionization rates are much larger even at very low plasma temperatures as compared to the ionization from the ground state. The overall neutral atom density is reduced by about a factor 5 as compared to the optical thin case in figure 6.

The resulting photon gas density is shown in figure 9. One can clearly see two bright spots near the strike point. 


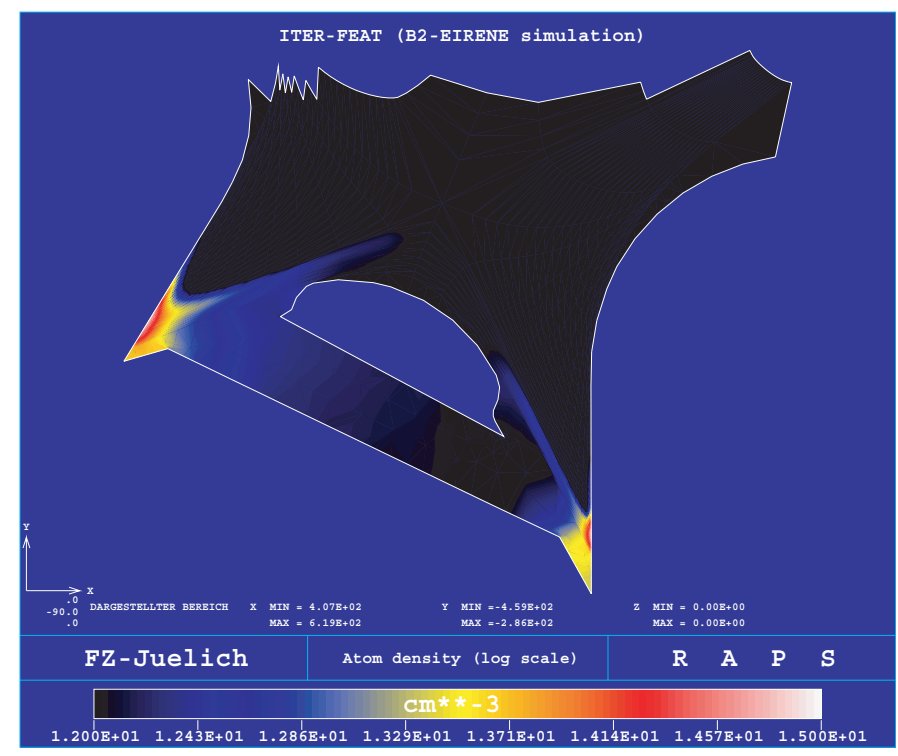

Figure 8. Neutral atom density, $\mathrm{cm}^{-3}$, log. colour scale. Same conditions as in figure 6, except for the self-consistent coupling to the Lyman $\alpha$ radiation field.

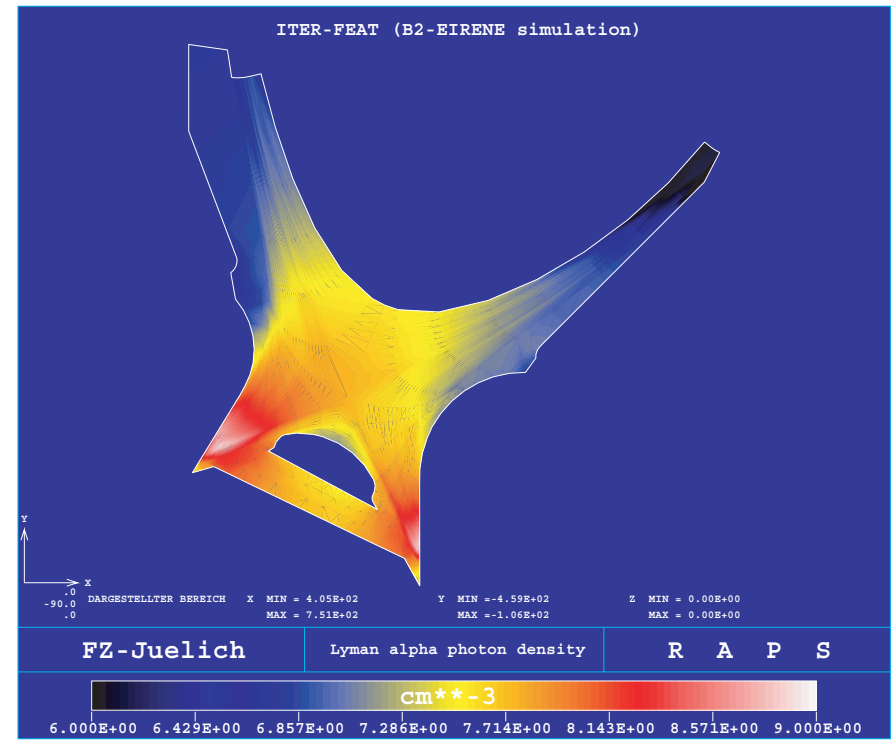

Figure 9. Same as figure 8, but Lyman $\alpha$ photon density rather than particle density. Clearly visible are two localized spots near the strike points.

The total emissivity of this line was $6 \times 10^{24}$ photons per second. About $50 \%$ of this flux was re-absorbed in the volume, $30 \%$ was absorbed at the vessel surfaces and $10 \%$ at the targets. About $10 \%$ of the total photon flux penetrates into the plasma core region not modelled here. (Note that photons need not obey particle conservation.) The photons re-absorbed in the SOL and divertor volume deposit their energy ( $10.2 \mathrm{eV}$ per photon) in the neutral atom cloud in the divertor and therefore lead to an enhanced degree of ionization. 
The total number of neutral atoms in the simulated edge plasma domain (SOL, divertor and vacuum region between the plasma and the walls) has decreased from about $10^{21}$ to $1.5 \times 10^{20}$ particles.

It is clearly shown that this internal iteration between the neutral gas and radiation field has lead to a significant modification of the population distribution, and hence, of the ionizationrecombination balance in the divertor and of the hydrogenic radiation losses to walls. This result is, of course, specific to the geometrical and physical conditions studied here and the consequences of radiation trapping on divertor dynamics have to be investigated on a case-to-case basis.

The response of the plasma on this new neutral particle conditions in the ITER-FEAT divertor has not been studied so far. It seems evident that in order to recover the initial divertor conditions (achieved with the optically thin assumption in reference [10]) some of the upstream boundary conditions between core and SOL plasma will have to modified. A quantitative statement about this crucial divertor design issue will be possible with the model extensions described in this paper.

The hitherto utilized zero-dimensional concept of population escape factors is certainly far too crude. The population escape factor defined in equation (8) is shown in figure 10. It is obvious that no single constant value can be assigned to the divertor region. We find a complicated pattern of regions, in which there is net emission, zero emission and even net absorption (negative population escape factors), respectively. Any particular fixed choice of that parameter in a plasma edge simulation for large and cold divertors is likely to introduce a significant additional uncertainty into one of the few components (neutral gas transport) of current divertor models, which are believed to be capable of a quantitative predictibility. In particular, the possibility of finding, locally, a negative population escape factor is a consequence of the non-local character of radiation transfer and certainly not accessible in simpler models.

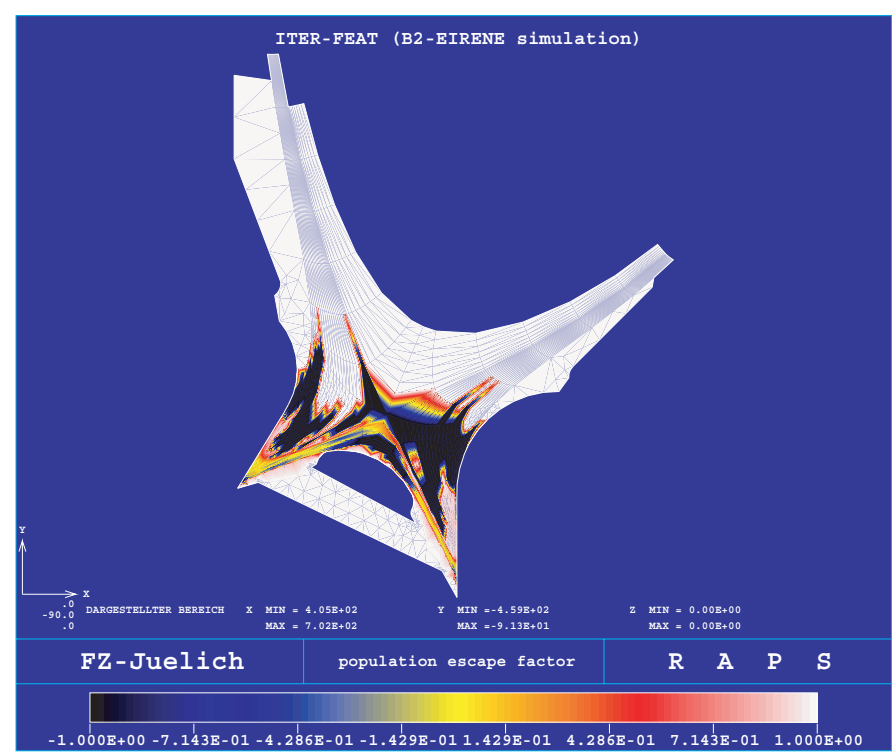

Figure 10. Population escape factor $\Theta_{p}$ profile (as defined in equation (8)). White colour indicates optically thin conditions locally, yellow colours indicate domains in which the optically thick limit applies (re-absorption of all Lyman $\alpha$ photons instantaneously), blue and black colours indicate domains with a net absorption, i.e. more absorption of photons originating from somewhere else in the plasma than local emission. 


\section{Conclusions and outlook}

A fully parallelized three-dimensional Monte Carlo neutral particle code for fusion edge plasma studies has been generalized to photon gas simulations (radiation transport). Doppler and Voigt broadened-line emission profiles have been implemented, absorption in the volume as well as diffuse and specular reflection at surfaces could be carried over from existing coding for neutral particles. The modified collision-radiative rate coefficients for transitions to and from the upper level of the Lyman $\alpha$ lines have been added to the atomic database for the EIRENE code. The code extensions have been validated against semi-analytical results (population escape factors) for idealized cases, up to quite high opacity (optical thickness of the order 30). Even transition to the optically dense blackbody radiation limit could be reproduced with high statistical precision. In first stand-alone applications, the Lyman $\alpha$ line radiation transport has been included in an B2-EIRENE study of ITER-FEAT divertor conditions. Typically $50 \%$ of this radiation is found to be re-absorbed in the volume, $1 / 3$ at the vessel and $10 \%$ at the divertor targets. The nonlinear response of absorption and emission on the neutral atom population and on the divertor hydrogen radiation, as well as on the ionization-recombination balance, has been investigated here with EIRENE. The response of the plasma conditions (in particular the electron temperature and density fields) within the context of a fully selfconsistent B2-EIRENE simulation still has to be studied. Further extensions of the photon gas transport model will, most importantly, also have to address Zeeman splitting, and the issue of partial redistribution. In the present paper, complete redistribution was assumed for the photon gas, and the standard partial re-distribution functions (termed 'scattering kernels' in this field of linear transport applications) have been used for the atomic and molecular components. The complete redistribution assumption for the photon gas will also have to be assessed in the future.

\section{References}

[1] Terry J L et al 1998 Phys. Plasmas 51759

[2] Adams M L et al 2001 J. Quant. Spectrosc. Radiat. Transfer. 71117

[3] Wan A S et al 1995 J. Nucl. Mat. 220-222 1102

[4] Behringer K 1998 Escape factors for line emission and population calculations MPI-Garching Report, IPP 10/11 and Atomic Data Analysis Structure (ADAS) (see http://adas.phys.strath.ac.uk)

[5] Marchand R et al 1992 Phys. Fluids B 4924

[6] Krasheninnikov S I et al 1988 Contrib. Plasma Phys. 28443

[7] Adams M L et al 2002 Contrib. Plasma Phys. 42395

[8] Reiter D et al The EIRENE code see http://www.eirene.de

[9] Feng Y et al 2002 Plasma Phys. Control. Fusion 44 611-25

[10] Kukushkin A S et al 2000 18th Fusion Energy Conf. (Oct. 2000) 2000 IAEA-CN-77, paper ITERP/10(R)

[11] Kukushkin A S 2002 Plasma Phys. Control. Fusion 44 931-43

[12] Cercignani C 1988 The Boltzmann Equation and Its Applications (Berlin: Springer)

[13] Mihalas D 1978 Stellar Atmospheres (San Francisco: Freeman)

[14] Modest M F 1993 Radiative Heat Transfer (New York: McGraw-Hill)

[15] Reiter D 1992 J. Nucl. Mat. 196-198 241

[16] Reiter D et al 1995 J. Nucl. Mat. 220-222 987

[17] Bates D R, Kingston A E and McWhirter R W P 1962 Proc. Roy. Soc. A 270155

[18] Irons F E 1979 J. Quant. Spectrosc. Radiat. Transfer. 221

[19] Wünderlich D and Fantz U 2001 A collisional-radiative model for $\mathrm{H}_{2}$ and $\mathrm{H}$ : extensions and applications MPI-Garching Report, IPP 10/17

[20] Sawada K and Fujimoto T 1995 J. Appl. Phys. 782913 